\title{
Energy Tunability in Laboratory 3D Nano-XRM
}

Jeff Gelb $^{1 *}$, David Vine ${ }^{1}$, Trevor Lancon ${ }^{2}$, Sylvia Lewis ${ }^{1}, \mathrm{SH} \mathrm{Lau}^{1}$ and Wenbing Yun ${ }^{1}$

1. Sigray, Inc., Concord, CA, USA.

2. Thermo Fisher Scientific, Houston, TX, USA

* Corresponding author: jgelb@sigray.com

Modern X-ray microscopy (XRM) has grown in popularity in recent years [1]. Owing to the high penetrating power of X-ray radiation and the promising resolution levels that can be achieved, XRM is now an established technique at many synchrotrons and become more commonplace in laboratory settings as well. Nano-XRM, in particular, is of interest due to its abilities to provide spatial resolutions in the $10 \mathrm{~s}$ to $100 \mathrm{~s}$ of nanometers [2]. While this technique is of considerable interest, still many researchers rely on precious synchrotron time in order to characterize their material systems.

One of the key benefits afforded by synchrotron radiation is the ability to fine-tune the incoming photon energy for each experiment. This is helpful for two key reasons:

1) The beam energy may be adjusted to just below an absorption edge of the material of interest, greatly enhancing the contrast of that material and, thus, producing higher-quality tomographic datasets.

2) By collecting images above and below a particular absorption edge, a material may be identified in a quasi-quantitative manner, aiding in speciation and providing a precise understanding of the composition within the microstructure.

Synchrotrons benefit researchers by providing precise control over the exact photon energy in use [3], which is one of the key benefits of being granted time on a nano-XRM beamline. While efforts have been made to migrate nano-XRM to laboratory environments, conventional laboratory nano-XRMs generally operate at a fixed energy, eliminating one of the critical aspects of XRM for materials research.

In our work, we have designed a nano-XRM with a small range of energy tunability, capitalizing on a novel microfocus laboratory X-ray source design. The X-ray source we have developed incorporates multiple target materials for establishing a range of spectra, with imaging successfully demonstrated at $2.7 \mathrm{keV}, 5.4 \mathrm{keV}, 6.4 \mathrm{keV}$, and $8.0 \mathrm{keV}$. While still far from the precise control offered by synchrotrons, this range of available energies grants researchers the opportunity to explore the benefits of multiple energies for both contrast enhancement and some degree of elemental discrimination. This source has been paired with a double-paraboloidal capillary condenser, providing exceptional illumination profiles and eliminating the off-axis aberrations encountered with conventional ellipsoidal optics, as well as a finely-structure Fresnel zone plate imaging objective for magnification. The result of these developments is a multi-energy 3D X-ray microscope with spatial resolution capabilities to $40 \mathrm{~nm}$ and fields of view up to $80 \mu \mathrm{m}$. Users may switch between energies via user-friendly software control, without the need for time-intensive optical realignments or costly system upgrades.

As an example of how this has been applied, we investigated a Nickle-Cobalt-Manganese (NCM) based cathode from a Lithium-ion battery across two energies: $5.4 \mathrm{keV}$ and $8.0 \mathrm{keV}$. The data were 
reconstructed via GPU-accelerated simultaneous iterative reconstruction technique (SIRT) [4], providing sufficient feature clarity for precise understanding of the material microstructure. We then developed an alignment and registration routine for the two scan results -5.4 and $8.0 \mathrm{keV}$ - which allowed the two datasets to be interrogated as a pair as well as on their own. While the absorption profile of Nickel follows the Lambert-Beer Law for attenuation from 5.4 to $8.0 \mathrm{keV}$, Cobalt and Manganese have absorption edges leading to nonlinearities between these two energies. Thus, by comparing the results, we are able to identify any regions of high Co or Mn concentration, as well as determine the optimal photon energy for enhancing the 3D imaging contrast of the composite material.

In this presentation, we will review the system design, imaging strategy, and experimental results from this multi-energy nano-XRM. We will also discuss how this system fits in to the context of multi-scale, multi-modal materials science, and the role of multi-energy nano-XRM in the context of correlative microscopy workflows.

\section{References:}

[1] P Withers, Advanced Materials \& Processing 170 (2012) p. 28-32.

[2] A Merkle and J Gelb, Microscopy Today 21 (2013) p. 10-15.

[3] PR Shearing, Y Wu, SJ Harris, NP Brandon, The Electrochemical Society Interface (2011) p. 43-47.

[4] D Gursoy, F De Carlo, X Xiao and C Jacobsen, Journal of Synchrotron Radiation 21 (2014) p. 11881193.

[5] The authors acknowledge Dr. Donal Finegan of National Renewable Energy Laboratory for providing the battery electrode samples and for many insightful discussions.
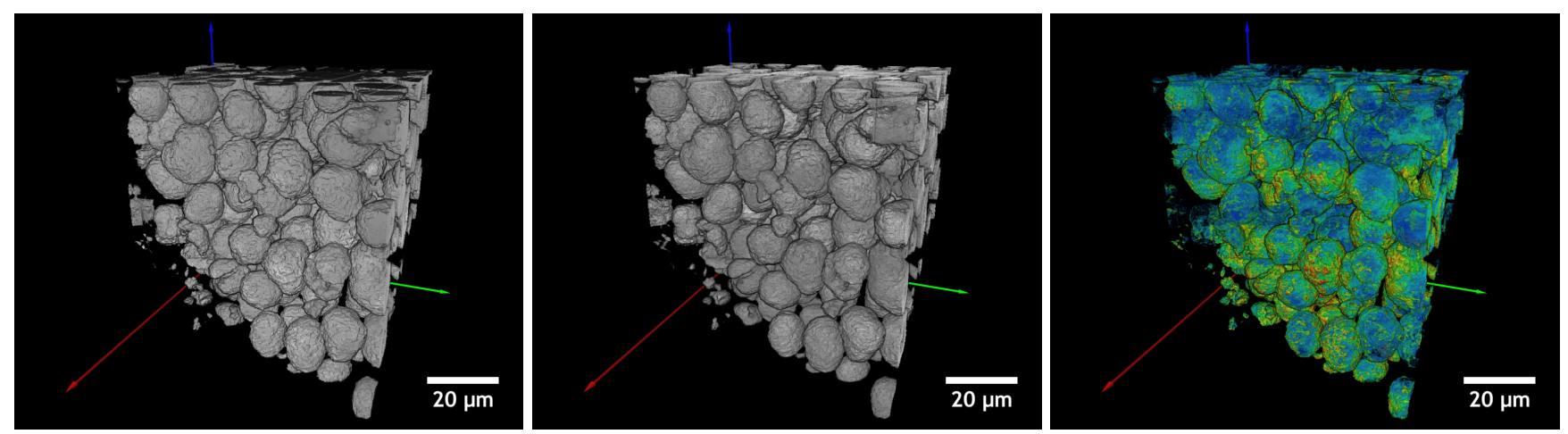

Figure 1. 3D renderings of the NCM battery electrode. The left image shows the specimen imaged at $5.4 \mathrm{keV}$, the middle shows the same ROI imaged at $8.0 \mathrm{keV}$, and the right image shows the difference of the two images rendered on a red-green-blue colormap. Blue shading indicates regions of similarity (i.e., absorption scaling by Lambert-Beer Law), while yellow/red shading indicates regions of difference (i.e., nonlinearity) between the two scans. 